Bull. Mater. Sci., Vol. 2, Number 4, November 1980, pp. 221-231. (C) Printed in India.

\title{
Physics of silver halides and their applications
}

\author{
D N BOSE and P A GOVINDACHARYULU* \\ Materials Science Centre, Indian Institute of Technology, Kharagpur 721 302, India \\ * Electrical Communication Engineering Department, Indian Institute of Science, \\ Bangalore 560 012, India
}

MS received 6 May 1980 ; revised 19 July 1980

\begin{abstract}
Silver halides are notable for exhibiting both ionic and electronic conductivity which is responsible for their widespread use in photography. Due to differences in ionicity and bonding, their properties vary from the highly ionic silver fluoride to the more covalent silver iodide. These fundamental properties such as defect formation energies, carrier mobilities and band-structures, particularly of silver iodide, have only recently been determined. One interesting finding is the presence of high surface fields, caused by differences between interstitial and vacancy formation energies, which results in separation of photo-generated electron hole pairs. This throws new light on the photographic process and on the suitability of silver bromide as a photographic material. $a-\mathrm{AgI}$ is also the first fast ion conductor to be discovered. Structural instability, low defect formation energy and optimum cation size are responsible for this phenomenon but existing models fail to explain all the experimental results. Recent microwave conductivity and neutron scattering experiments which provide fresh insight are discussed.
\end{abstract}

Keywords. Silver halides; ionic conductivity ; defect energy ; carrier mobility ; surface field; photography; fast ion conduction.

\section{Introduction}

Although silver halides have ling played an important role in the devel spment of our understanding of crystclline solids, some of their fund.mental properties are still in the process of being unravelled. Often overshsd swed by the more famous group of alkali halides, the silver halides have nevertheless also been model solids for experimenting and testing theories, speci.lly of the defect solid state (Brown 1967). The silver salts are notable for their unique combination of ionic as well as electronic conductivity which has given them their prominence in photography (Neblette 1964). Another intriguing phenomenon first discovered in silver iodide, fast ion conduction (Mahan and Roth 1976) is of great theoretical interest and is finding applications in solid state batteries. This paper will attempt to relate some of the fundamental ionic and electronic properties of these halides, as found recently, with these applications. 


\section{Structure}

There are interesting similarities as well as differences in the family of silver halides starting with their crystal structure. While silver fluoride, chloride and bromide all possess the rock-salt structure (figure 1a) with ions having a 6-fold coordination, silver iodide in its stable $\beta$-phase has the hexagonal wurzite structure with 4 -fold coordination (figure 1b). Earlier controversy has been resolved with the proof

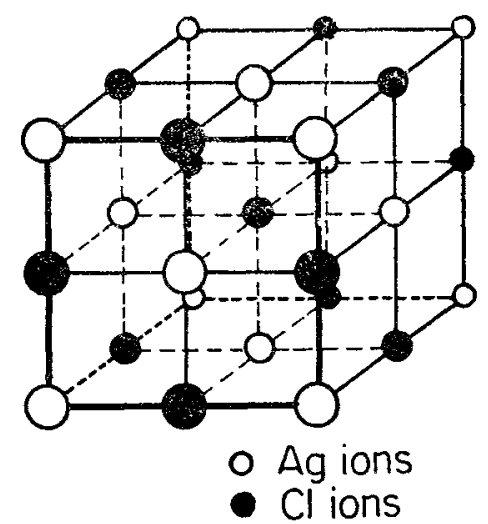

(a)

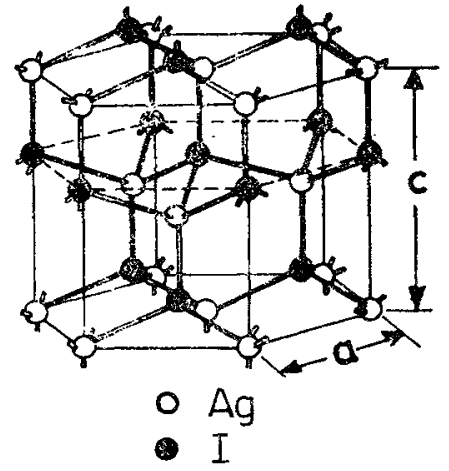

(b)

Figure 1. (a) Crystal structure of AgCl. (b) Crystal structure of AgI.

that the $\gamma$-phase of AgI which has the zinc-blende structure closely related to the wurzite structure and with the same coordination number, is meta-stable. At a pressure of $4 \mathrm{~K}$ bars $\beta$-AgI transforms into the rock-salt structure. At a temperature of $147^{\circ} \mathrm{C}$ it undergoes a phase transformation into $a$-AgI, the first fast ion conductor to be discovered, in which the iodine atoms occupy a b.c.c. structure while the two silver ions, being disordered and statistically distributed among 12 stable sites, are responsible for the high ionic conductivity.

The difference in structure between the silver halides has been explained very successfully by the theory of ionicity and bonding (Phillips 1973). This predicts a 6-fold coordination for ionicity greater than 0.785 on the Phillips scale and a 4-fold coordination for lower ionicity and greater covalence. It has been shown that the ionicity of $\mathrm{AgCl}, \mathrm{AgBr}$ and $\mathrm{AgL}$ are $0.856,0.850$ and 0.770 respectively, this explaining the difference in crystal structures and providing a good test of the theory (table 1).

\section{Ionic conductivity}

The classical experiments of Tubandt in 1913 revealed that silver iodide exhibited cationic conductivity. Since then it has been establisbed that just as alkali halides exhibit Sch ottky-type disorder, the silver halides have predominantly Frenkel defects, comprising of interstitial silver ions and silver ion vacancies. It is the optimum size of the silver ion and its electronic structure which is responsible for its high 
Table 1. Structure and defect properties of silver halides.

\begin{tabular}{lllllllll}
\hline $\begin{array}{l}\text { Com- } \\
\text { pound }\end{array}$ & Ionicity & Structure & $\begin{array}{c}h_{F} \\
(\mathrm{eV})\end{array}$ & $\begin{array}{c}W_{i} \\
(\mathrm{eV})\end{array}$ & $\begin{array}{c}W_{v} \\
(\mathrm{eV})\end{array}$ & $\begin{array}{c}e \phi \\
(\mathrm{eV})\end{array}$ & $\begin{array}{c}h_{m}^{+} \\
(\mathrm{eV})\end{array}$ & $\begin{array}{c}h_{m}^{-} \\
(\mathrm{eV})\end{array}$ \\
\hline $\mathrm{AgCl}$ & 0.856 & Rock-salt & 1.44 & 0.97 & 0.47 & -0.25 & 0.145 & 0.350 \\
$\mathrm{AgBr}$ & 0.850 & Rock-salt & 1.06 & 0.21 & 0.85 & 0.32 & 0.149 & 0.384 \\
$\mathrm{AgI}$ & 0.770 & Wurzite & 0.64 & 0.20 & 0.44 & 0.12 & $\begin{array}{c}0.41,0.38 \\
(\| c)(\| a)\end{array}$ & $\begin{array}{c}0.500 .52 \\
(\| c)(\| a\end{array}$ \\
\end{tabular}

mobility. Measurements of ionic conductivity over a wide range of temperatures and with different dopants have been used for determining the defect formation and migration energies (Barr and Lidiard 1970). As is well-known, at relatively high temperatures, the conductivity is given by

$$
\sigma T=A_{1} \exp \left(-E_{1} / k T\right) \text {. }
$$

When conduction is due to one type of charge carrier,

$$
E_{1}=\left(h_{F}+h_{m}\right) / 2 \text {, }
$$

where $h_{F}$ is the formation energy of the Frenkel defect and $h_{m}$ is the migration energy of the interstitial and $A_{1}$ is a constant dependent on the jump distance and vibrational frequency.

At lower temperatures where conductivity is determined by frozen-in defects already present,

$$
\sigma T=A_{2} \exp \left(-E_{2} / k T\right)
$$

where $E_{2}=h_{m}$ is the migration energy of the mobile defect already present. At still lower temperatures, an activation energy $E=h_{m}+\frac{1}{2} h_{a}$, where $h_{a}$ is the association energy of defects, may occur but will not be discussed further here.

Such measurements had been used to find $h_{F}$ and $h_{m}$ for $\mathrm{AgCl}$ and $\mathrm{AgBr}$ as given in table 1 and found in many texts in solid-state physics (Brown 1967). Here $h_{m}^{+}$and $h_{m}^{-}$are the activation energies of migration of positive and negative ions respectively. Theie are determined, for example, by introducing divalent cations to replace monovalent silver which thus increases the concentration of negatively charged vacancies. Calculations have also been carried out to determine $h_{F}$ and $h_{m}$ assuming various microscopic models for ionic motion. A comparison between theoretical and experimental values of defect formation and migration energies for $\mathrm{AgCl}$ and also experimental values for $\mathrm{AgBr}$ (Friauf 1962) are given in table 2. It is seen that for $h_{F}$ there is reasonable agreement between thecry and experiment in the case of $\mathrm{AgCl}$. It is also evident that the collinear interstitialcy jump meshanism has by far the lowest activation energy for both $\mathrm{AgCl}$ and $\mathrm{AgBr}$ while direct interstitial jump requires prohibitively high energies. It has thus been possible to establish by comparison with the results of diffusion 
Table 2. Comparison between theoretical and experimental defect energies.

\begin{tabular}{lccc}
\hline $\begin{array}{c}\text { Formation and migration } \\
\text { energies (eV) }\end{array}$ & $\begin{array}{c}\mathrm{AgCl} \\
(\text { Expt.) }\end{array}$ & $\begin{array}{c}\mathrm{AgCl} \\
\text { (Theory) }\end{array}$ & $\begin{array}{c}\mathrm{AgBr} \\
\text { (Expt.) }\end{array}$ \\
\hline Frenkel defect formation $\left(h_{F}\right)$ & 1.69 & 1.76 & 1.27 \\
Vacancy migration $\left(h_{m}^{-}\right)$ & 0.33 & 1.2 & 0.36 \\
Interstitialcy (average) & 0.15 & & 0.15 \\
Collinear interstitialcy & & -0.1 to 0.4 & 0.078 \\
Non-collinear interstitialcy & & 0.8 & 0.23 \\
Direct interstitial & & 3.0 & \\
\hline
\end{tabular}

experiments that the silver ions move by interstitialcy or indirect interstitial mechanism and not by direst jumps between interstitial sites. Such calculations have yet to be carried out for AgI.

Comparable experimental data on AgI were also contradictory and scarce. One reason is that the phase transition at $147^{\circ} \mathrm{C}$ made it difficult to grow quality single crystals from the melt as for $\mathrm{AgCl}$ and $\mathrm{AgBr}$. Further, results on polycrystalline AgI were ambiguous due to the coexistence of the $\beta$ and $\gamma$ phases in varying proportions, depending on the method of preparation.

The ionic conductivity in the anisotropic $\beta$-phase posed an interesting problem. In a hexagonal crystal there will be two independent components of conductivity, along and perpendicular to the $c$-axis. Thus the activation energies will be $E_{10}$, $E_{20}$ and $E_{1 a}$ and $E_{2 a}$ which could all be different. However, $h_{F}$, the Frenkel defect formation energy is expected to be a bulk property independent of direction of measurement. Thus it is expected from equations (1) and (2) that $h_{F}=2\left(E_{10}-E_{2 c}\right)$ $=\left(E_{1 a}-E_{2 a}\right)$. However Cochrane and Fletcher (1971) who first performed detailed anisotropy experiments found that this did not follow from their results.

The problem of single crystal growth was partly solved by using the gel rethod near room temperature. Comparatively small pyramids and platelets were grown by Cochrane and Fletcher, Suri and later by Govindacharyulu and Bose. However the $c$ dimension was always much less than the other dimensions and it was realised that non-uniform space-charge effects may have an important effect on the measurements. Although eleatronic space-charge is common place in semiconductors, ionic space-ch irge is also readily observable in insulating polar crystals and has been discussed theoretically by Kliewer (1966). It arises from the fact that at the surface of such a crystal positive and negatively charged defects, interstitials and vacancies can and do occur separately and not necessarily in pairs. Thus the Frenkel defect energy can be divided into $h_{F}=h_{i}+h_{n}$, the energies of interstitial and vacancy formation respectively. The one with higher formation energy will predominate at the surface as fixed charge with a compensating distri- 
buted space-charge region of the opposite type following immediately below. Thus for crystals thin in the $c$ direction, measurements along the $a$ direction would give $E_{1 a}-E_{2 a}=h_{\mathrm{a}}$ if $h_{i}<h_{\mathrm{v}}$. The fact that positively charged species dominated at the surface space-charge region was shown by Bardeen-Brattain type ambient experiments with ozone and water-vapour. It was thus possible for the first time (Govindacharyulu et al 1978), using the anisotropy of AgI, to determine that the interstitial and vacancy formation energies are $h_{\mathrm{t}}=0.20 \mathrm{eV}$ and $h_{v}=0.44 \mathrm{eV}$ respectively giving $h_{F}=h_{\imath}+h_{v}=0.64 \mathrm{eV}$. Values for $\mathrm{AgCl}$ and $\mathrm{AgBr}$ are given in table 1 for comparison, but have been determined in a different manner using measurements on thin films.

From the values of $h_{F}$ for $\mathrm{AgBr}$ and $\mathrm{AgI}$ (table 1) it follows that the concentration of Frenkel defects at $300^{\circ} \mathrm{K}$ is much higher in AgI than in AgBr, $2.9 \times$ $10^{18} / \mathrm{cm}^{3}$ compared to $8 \times 10^{14} / \mathrm{cm}^{3}$. However, due to much lower $h_{m}^{+}$, ionic mobility is much lower in AgI, $4.0 \times 10^{-7} \mathrm{~cm}^{2} / \mathrm{V}$ sec for silver interstitials compared to $4 \times 10^{-3} \mathrm{~cm}^{2} / \mathrm{V}$ sec for $\mathrm{AgBr}$.

\section{Surface properties}

An immediate consequence of the surface space-charge in ionic crystals is that considerable fields are present and the surface potential, which deviates from the bulk, is given by $e \phi=\frac{1}{2}\left(W_{\mathrm{b}}-W_{i}\right)$. Impurities may thus segregate at the surface depending on their charge as found recently by Baetzold (1975). Values of surface potential for the three common halides are also given in table 1 . It is apparent that (i) surface fields are largest in $\mathrm{AgBr}$ and the fixed negative surface charge is neutralised by a positive space-charge region of interstitials, the net field being such as that to impel electrons into the bulk while holes are trapped at the surface and (ii) due to increased band-bending the net concentration of interstitials at the surface is at least 2 orders of magnitude larger in $\mathrm{AgBr}$ than in AgI. These conclusions have important implications for the photographic process.

\section{Electronic and optical properties}

Distinguishing between electronic and ionic conductivity is a long-standing problem in many solids including silver halides. Ionic conduction involves mass transfer and hence correlation with diffusion studies has been useful in determining ionic properties. Electronic conductivity in these materials cannot be studied by conventional resistivity, Hall effect and mobility measurements due to ionic polarisation and charge motion, which reduces the field within the solid setting up fields only across the electrodes. Two methods have been used to circumvent this problem: (i) Wagner cell measurements (Wagner and Wagner 1957) utilising an asymmetric d.c. cell with one reversible and one blocking contact such as $\mathrm{Ag} /$ $\mathrm{AgI} / \mathrm{C}$. The steady-state current-voltage relation can be used to determine the electronic (or hole) component of conduction in a predominantly ionic conductor; (ii) pulsed field measurements in which the duration of the applied field is less than the time required for ionic polaristion to develop. This technique was developed by Brown at the University of Illinois to measure drift mobility in $\mathrm{AgCl}$ and $\mathrm{AgBr}$ and later used by Govindacharyulu and Bose (1977) to determine drift mobility of holes in $\beta$-AgI. The results are given in table 3 . 
Table 3. Mobility of charge carriers and band-gaps in silver halides.

\begin{tabular}{|c|c|c|c|c|c|c|c|c|}
\hline \multirow{2}{*}{$\begin{array}{l}\text { Com- } \\
\text { pound }\end{array}$} & \multirow{2}{*}{$\mu_{n_{e}}$} & \multirow{2}{*}{$\mu_{p c}$} & \multirow{2}{*}{$\mu_{n g}$} & \multirow{2}{*}{$\mu_{\boldsymbol{p}_{j}}$} & \multirow{2}{*}{$\mu_{A q}+$} & \multirow{2}{*}{$\mu_{A D^{-}}$} & \multicolumn{2}{|c|}{$\mathrm{E}_{g}(\mathrm{eV})$} \\
\hline & & & & & & & direct & Indirect \\
\hline $\mathrm{AgCl}$ & 50 & $2 \times 10^{-4}$ & & & $5 \times 10^{-3}$ & $6 \times 10^{-6}$ & $5 \cdot 15$ & $3 \cdot 25$ \\
\hline $\mathrm{AgBr}$ & 75 & 1 & $2 \times 10^{-1}$ & $10^{-3}$ & $4 \times 10^{-3}$ & $4 \times 10^{-8}$ & $4 \cdot 29$ & $2 \cdot 69$ \\
\hline $\mathbf{A} \dot{\mathbf{g} I}$ & 30 & 12 & & & $\begin{array}{c}4 \times 10^{-7} \\
(\| c)\end{array}$ & $\begin{array}{c}2 \times 1^{-8} \\
(\| c)\end{array}$ & $2 \cdot 83$ & \\
\hline
\end{tabular}

$\mu_{n_{e}}, \mu_{p_{0}}$ electron and hole mobilities in crystals. $\mu_{n_{g}}, \mu_{p_{g}}$ elcctron and hole mobilities in grains. $\mu_{A g^{+}}, \mu_{A g^{-}}$interstitial and vacancy mobilities in crystals. All values in $\mathrm{cm}^{2} / \mathrm{Vsec}$ at $300^{\circ} \mathrm{K}$.

In the experiment shown in figure $2 \mathrm{a}$ a pulse of absorbed light or electrons is incident on one surface of a cyrstal while a d.c. field is applied across it in synchronism. The arrival of the generated carriers at the second electrode is measured on an oscilloscope as in the well-known Shockley-Haynes drift mobility experiment. The drift mobility is given by $\mu=L^{2} / V \tau_{t}$, where $L$ is the length of the crystal, $V$ is the applied voltage and $\tau_{t}$ is the transit time of the carrier. One notable distinction between the Shockley-Haynes and the Brown experiments is that while only the minority carrier mobility can be measured by the first method in the latter, the

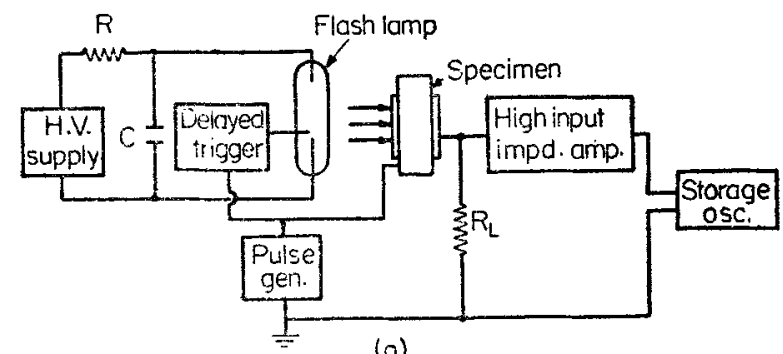

(a)

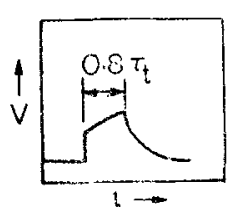

(b)

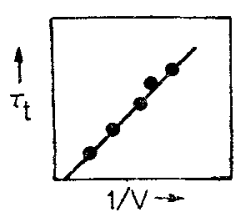

(c)

Figure 2. (a) Experiment for drift mobility measurement in ionic materials. (b) Output voltage vs, time, giving transit time. (c) Transit time $\tau_{\ddagger}$ vs. $1 / V$ where $V=$ applied drift voltage. 
drift mobility of both types of carriers can be measured by merely changing the polarity of the applied drift field. This is because in this case the transit-time is less than the dielectric relaxation time and space-charge neutrality conditions are not maintained. In $\beta$-AgI for example, the drift mobility of electrons and boles at $300^{\circ} \mathrm{K}$ have thus been found to be $30 \mathrm{~cm}^{2} / \mathrm{V}$ sec and $12 \mathrm{~cm} / \mathrm{V}$ sec respectively (Bose and Govindacharyulu 1976). In all the silver haljdes the mobility is controlled by shallow traps at lower temperatures, the effective mobility decreasing with decrease in temperature due to carriers remaining immobilised at these lccalised states. The identity of these traps is a subject for continuing investigation. At higher temperatures the mobility goes through a maximum and then decreases as usual due to phonon scattering.

Of crucial importance as far as optical properties are concerned is the nature of the electronic band-structure. In semiconductor devices it has been iealised over the last 20 years that the operation of devices such as lasers, LEDs or Gunn diodes is directly dependent on the band-structure of a meterial. As is well known materials can be divided into direct band-gap materials like GaAs, InSb, $\mathrm{CdS}$ for which the minimum of the conduction as well as the maximum of the valence band have the wave vector $k=0$ (figure 3a) and (ii) indirect band-gap materials like silicon and germanium (figure $3 \mathrm{c}$ ) in which the conduction band minimum is displaced from $k=0$. Optical absorption and emission involve vertical electronic transition with no change in $k$ vector since a photon has negligible momentum on this scale. Thus while direct band-gap materials have high probability of absorption or emission of radiation, indirect band-gap materials require the participation of a phonon which is either absorbed or emitted, to conserve momentum.

It had been shown by Bassani et al (1965) from band-structure calculations that $\mathrm{AgCl}$ and $\mathrm{AgBr}$ represent another type of indirect band-gap material (figure $3 \mathrm{~b}$ ) in which the maximum of the valence band is displaced from $k=0$ although the minimum of the conduction band is still at this position. However, considerable controversy existed as to the nature of the band-gap in $\beta$-AgI. Govindacharyulu and Bose (1979) have recently shown by measurements of photo-conductivity excitation spectra and anisotropy that $\beta$ - $\mathrm{AgI}$ is a direct band-gap material very much like $\mathrm{CdS}$ which it resembles in structure. This is supported by pseudo-

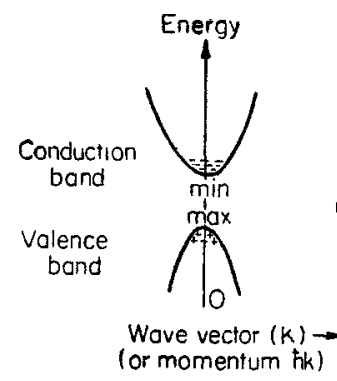

(a)

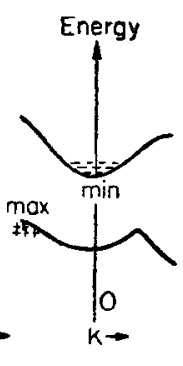

(b)

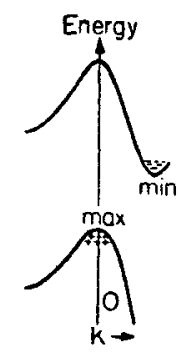

(c)

Figure 3. Energy band diagram of three types of semiconductors. (a) Direct band gap like GaAs, InSb, AgI, etc. (b) Indirect band gap like $\mathrm{AgCl}, \mathrm{AgBr}$, etc. (c) Indirect band gap like $\mathrm{Si}, \mathrm{Ge}, \mathrm{GaP}$, etc. 
potential calculations of band-structure by Smith (1976). Thus while the recombination of excess carriers in $\beta$-AgI will be rapid, it will be comparatively slow in $\mathrm{AgCl}$ and $\mathrm{AgBr}$. In fact the anomaly of $\mathrm{AgBr}$ having a smaller band-gap of $2.69 \mathrm{eV}$ compared with $2.83 \mathrm{eV}$ of $\mathrm{AgI}$ is now explained, since the former is indirect while the latter is direct, and the direct band-gaps are found to scale according to Welker's rule. It is thus expected that AgI could be made to exhibit stimulated emission at low temperatures. The study of electron-hole droplet formation in polar materials is also of current interest and the theory suggests the possibilities of stable droplets in AgBr (Beni and Rice 1978) while AgI is yet to be examined.

The peculiar valence band structure of the silver halides is due to the close proximity of bands originating on the halogen $p$ levels and the silver $d$ states. Because the rock salt structure of $\mathrm{AgCl}$ and $\mathrm{AgBr}$ has inversion symmetry, these states do not mix at $\Gamma$, the centre of the Brillouin zone, but away from $\Gamma$ the repelling of bands causes the uppermost levels to bend upwards rapidly. This leads to widely separated direct and indirect gaps. The wurzite structure of $\beta$ AgI without inversion symmetry does not force the electronic states to form an indirect edge in $\beta$-AgI which is thus unique among the silver halides. This is borne out by the photoemission experiments of Bauer and Spicer (1976). The detailed band-structures are given in figure 4.

\section{Photographic properties}

Interestingly enough the first photosensitive material used in the famous Daguerrotype process of 1839 by Louis Daguerre was silver iodide. It was discarded for AgBr shortly after because of the latter's higher sensitivity and wider spectral response. Although microscopic theories of the photographic process were developed by Gurney and Mott (1938) to be modified by Mitchell in 1946, the difference in material properties was not understocd until recently. Wei and Brown (1973) remarked on the fortuitous combination of elecironic and ionic

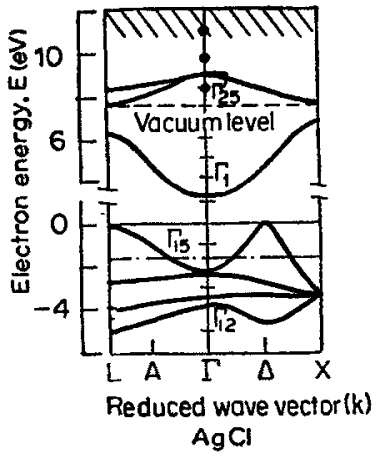

(a)

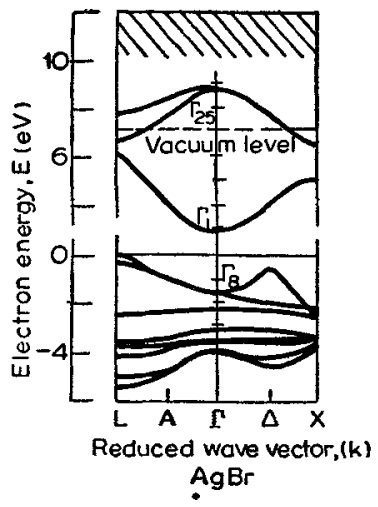

(b)

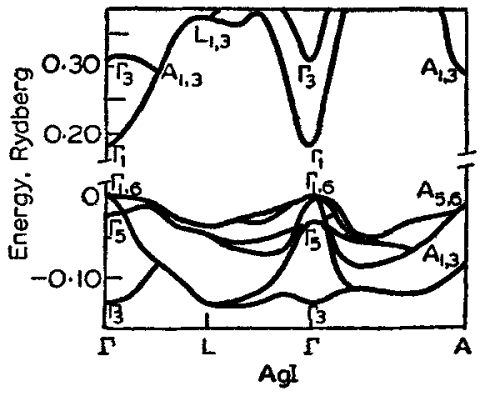

(c)

Figure 4, (a) Band-structure of $\mathrm{AgCl}$. (b) Band-structure of $\mathrm{AgBr}$. (c) Bandstructure of AgI. 
properties which thake silver bromide still pre-ominent among photographis materials.

According to the Mott-Gurney theory when a photon is incident on silver bromide, it is aborbed if its energy $h v>E_{g}$, the band-gap of the semiconductor, ereating an electron-hole pair. The spectral response of pure $\mathrm{AgBr}$ extends only to wavelength of $0.46 \mu \mathrm{m}$ and is widened by using suitable sensitising dyes. The hole is trapped near the surface of the grain which is typically 0.02 to $4 \mu$ in size, while the electron migrates into the grain aided by the surface field to be trapped by a previously neutral electron trap called a sensitivity speck. This may consist of a sulphur atom. Being charged negatively on acquiring an electron, the speok attracts a mobile positive charge, which, ir $\mathrm{AgBr}$, is a positive silver ion. The speck thus collects a neutral silver atom which forms a latent image capable of being developed. In practice it is found that about 4 photons are necessary to create a developable latent image.

The ifnportant differences between silver bromide and iodide in the photographic process are the following (i) the indirect band-gap of the former reduoes the probability of direct electron-hole recombination (ii) higher surface field in $\mathrm{AgBr}$ which aids electron motion, (iii) lower activation energy for migration $0.14 \mathrm{eV}$ for AgBr compared to $0.38-0.41 \mathrm{eV}$ for AgI leads to much higher silvir interstitial mobility, $4 \times 10^{-3} \mathrm{~cm}^{2} / \mathrm{V}$ sec and $4 \times 10^{-7} \mathrm{~cm}^{2} / \mathrm{Vsec}$ respectively, and (iv) the difference in hole mobility $\mu_{P_{e}}, 1 \mathrm{~cm}^{2} / \mathrm{V} \mathrm{sec}$ for $\mathrm{AgBr}$ compared with $12 \mathrm{~cm}^{2} / \mathrm{V}$ sec for AgI in large single crystals as given in table 3, the ratio being similar in emulsion grains. Since $h_{F}=0.64 \mathrm{eV}$ for AgI compared with $1.06 \mathrm{eV}$ for $\mathrm{AgBr}$, the concentration of Frenkel defects at $300^{\circ} \mathrm{K}$ are $2.9 \times 10^{18} / \mathrm{cm}^{8}$ and $8 \times 10^{14} / \mathrm{cm}^{3}$ respectively. Assuming uniform distribution of these defects and the interstitial mobilities mentioned above, the time for capture of an interstitial if $\mathrm{AgI}$ and $\mathrm{AgBr}$ are $5.7 \times 10^{-4} \mathrm{sec}$ and $2.7 \times 10^{-6} \mathrm{sec}$ respectively for an electric field of $10^{3} \mathrm{~V} / \mathrm{cm}$. Thus interstitial capture by trapped electrons is favoured in $\mathrm{AgBr}$ while hole capture is more probable in AgI. The serendipitous combination of an indirect gap which necessitates the participation of a phonon in recombination, the asymmetry between electron and hole mobilities and the high intetstitial silver ion mobility transpire to give $\mathrm{AgBr}$ its unique photographice properties.

\section{Fast ion conduction}

As mentioned earlier $\beta$-AgI undergoes a phase transition at $147^{\circ} \mathrm{C}$ into ald which is a fast ion conductor. $\mathrm{AgCl}$ and $\mathrm{AgBr}$ also undergo similar transition at $455^{\circ} \mathrm{C}$ and $425^{\circ} \mathrm{C}$ respectively. The high temperature phases have conductivities of the order of $1(\Omega \mathrm{cm})^{-1}$, as high as liquid electrolytes. At the $\beta \rightarrow a$ tratisition of AgI the conductivity increases by more than 3 orders of magnitude, and remains almost constant decreasing somewhat on melting. The activation entrgy in $a$ AgI is only $0.10 \mathrm{eV}$ and is typical of a series of derived compounds such as $\mathbf{R b}$ $\mathrm{Ag}_{4} \mathrm{I}_{5}$ which is a fast ion conductor at $300^{\circ} \mathrm{K}$. $\mathrm{CuBr}$ and $\mathrm{CuI}$ also display dimilar transitions and fast ion conduction in the high temperature phase. The phenomenon has been reviewed in detail recently (Mahan and Roth 1976) and hence 
will only be briefly discussed here. Theoretical models used have been (i) hopping conduction (ii) cooperative interaction model and (iii) free ion like model but none have satifactorily explained all the experimental data. For example, all hopping conduction models predict an increase in conductivity with frequency unlike the decrease observed experimentally for $a+\mathrm{AgI}$ at microwave frequencies by Funke (1976). The free-ion like model prediats a decrease with frequency but cannot explain conductivity poaks obtained in the microwave range.

An interesting calculation based on motion of cations along tunnels in the $\{100\rangle$ direction of the b.c.c. anion lattice of a-AgI has been developed by Flygare and Huggins (1973). The activation energy for motion has been caleulated as a function of cationic size and shows a minimum for cations of radii $0.83 \AA$ which is very close to the radius of the higly mobile silver ion. Smaller ions face larger energy barriers due to electrostatic attraction while larger cations are hindered by the repulsive term in potential energy.

Two experimental techniques that have provided interesting insight are the microwave measurements of Funke (1976) which show that the jump distances are of the order of $5 \AA$ and that the mean time of flight is of the same order as residence time at sites $\simeq 10^{-11} \mathrm{sec}$. Thus cationic motion in AgI in intermediate between diffusion and hopping mechanisms.

The other technique is neutron soattering to determine the lattico dynamics of $\beta$ and $a$-AgI. The results of Buhrer et al (1978) show the presence of very low lying dispersionless $T_{0}$ modes. These provide a strong contribution to thermal motion because they have a high density of states. The r.m.s. displacement of $\mathrm{Ag}$ ions is thus quite large increasing from $0.164 \AA$ in $\mathrm{AgCl}, 0.173 \AA$ in $\mathrm{AgBr}$ to $0.292 \AA$ in $\mathrm{AgI}$, thus favouring promotion of a cation to an interstitial site. However no mode softening has been observed between $80^{\circ} \mathrm{K}$ and $295^{\circ} \mathrm{K}$ and the zone-centre low frequency mode is stable up to the transition point. The phase transition thus appears to be driven by the entropy change due to cation disordering, the transition temperature being given by $T_{c}=\Delta H / \triangle S$, where $\triangle H$ is the change in enthalpy.

A number of solids related to AgI also exhibit similar properties, among them $\mathrm{Ag}_{3} \mathrm{SI}, \mathrm{Ag}_{2} \mathrm{HgI}_{4}$. The most interesting from the point of view of applioations as solid elctrolytes in batteries (Funke 1976) are the group of isostructural compounds $\mathrm{MAg}_{4} \mathrm{I}_{5}$ where $\left(M=\mathrm{NH}_{4}, \mathrm{~K}\right.$ or $\left.\mathrm{Rb}\right)$. These compounds are virtually pure ionic conductors at room temperature with conductivities as high as $0.2 \Omega^{-1} \mathrm{~cm}^{-1}$ and have been used in cells such as $\mathrm{Ag} / \mathrm{RbAg}_{4} \mathrm{I}_{5} / \mathrm{RbI}_{3}, \mathrm{C}$. The main drawback of these batteries is their low output voltage of $0.6 \mathrm{~V}$ and hence low energy densities. The search is thus continuing for better materials such as those based on lithium ion conductors.

It is thus evident that the fundamental properties now being determined such as band-structure, defect formation and migration energies of silver halides are of considerable importance in determining their applications. Better understanding of this relatively simple class of solids should thus help the development of new materials, for example less expensive ones for silver-less photography in the infrarod region and other materials for high-energy density solid-state batteries. 


\section{References}

Baetzold R C 1975 Appl. Phys. Lett. 12709

Barr L W and Lidiard A B 1970 Physical Chemistry 10152

Bassani F, Knox R S and Fowler W B 1965 Phys. Rev. 137 A1217

Bauer R S and Spicer W E 1976 Phys. Rev. B14 10, 4539

Beni G and Rice T M 1978 Phys. Rev. B18 2, 768

Bose D N and Govindacharyulu P A 1976 Physics of Semiconductors (ed.) F G Fumi (Amsterdam : North Holland) p. 1180

Brown F C 1967 Physics of Solids (New York : W A Benjamin, Inc.)

Buhrer W, Nicklow R M and Bruesch P 1978 Phys. Rev. B17 8, 3362

Cochrane G and Fletcher N H 1971 J. Phys. Chem. Solids 322557

Flygare W H and Huggins R A 1973 J. Phys. Chem. Solids 341199

Friauf R T 1962 J. Phys. Chem. Solids 662376

Funke K 1976 Superionic Conductors (New York: Plenum Pub. Co.)

Funke K 1976 Prog. in S.S. Chemistry 11345

Govindacharyulu P A and Bose D N 1977 J. Appl. Phys. 48 3, 1981

Govindacharyulu P A, Bose D N and Suri S K 1978 J. Phys. Chem. Solids 39961

Govindacharyulu P A and Bose D N 1979 Phys. Rev. 1376532

Gurney R W and Mott N F 1938 Proc. R. Soc. A164 151; 1964 Electronic processes in ionic crystals (Dover: New York)

Kliewer K L 1966 J. Phys. Chem. Solids 27 705, 719

Liang C C 1974 "Solid State Batteries," Appl. Sol. St. Science 495

Mahan G D and Roth W L (eds.) 1976 Superionic Conductors (New York : Plenum)

Neblette C B 1964 Photography (New York : Van Nostrand)

Phillips J C 1973 Bonds and bands in semiconductors (New York : Academic Press)

Smith P V 1976 J. Phys. Chem. Solids p. 37765

Wagner J B and Wagner C 1957 J. Chem. Phys. 261597

Wei J S and Brown F C 1973 Photog. Sci. and Engg. 17 2, 197 